\title{
MicroRNA-126 suppresses the proliferation and migration of endothelial cells in experimental diabetic retinopathy by targeting polo-like kinase 4
}

\author{
YARONG ZHENG ${ }^{1,2^{*}}$, YI LIU $^{1,2^{*}}$, LILI WANG $^{1,2^{*}}$, HANCHUN XU ${ }^{1,2}$, \\ ZHIQING LU ${ }^{1,2}$, YALING XUAN ${ }^{1,2}$, WENJING MENG ${ }^{1,2}$, LIN YE ${ }^{1,2}$, DEJIA FANG ${ }^{1,2}$, \\ YEKAI ZHOU ${ }^{1,2}$, KUNMAO KE ${ }^{1,2}$, YANLI LIU ${ }^{1,2}$ and MEIXIA AN ${ }^{1,2}$ \\ ${ }^{1}$ Department of Ophthalmology, The Third Affiliated Hospital of Southern Medical University; \\ ${ }^{2}$ Guangdong Provincial Key Laboratory of Bone and Joint Degeneration Diseases, Guangzhou, Guangdong 510630, P.R. China
}

Received April 25, 2020; Accepted October 13, 2020

DOI: $10.3892 /$ ijmm.2020.4775

\begin{abstract}
As a specific microvascular complication of diabetes, diabetic retinopathy (DR) causes severe visual impairment in patients with diabetes. The expression of microRNA-126 (miRNA/miR-126) has previously been found to be significantly decreased in the serum of patients with DR. In the present study, the functions of miR-126 and its mechanisms of action in experimental diabetic retinopathy were examined in rats with streptozotocin (STZ)-induced diabetes and in high glucose (HG)-induced human retinal capillary endothelial cells (HRCECs). In vivo, diabetic rat models were established and the rats were intravitreally injected with lentivirus expressing rno-miR-126 (lenti-miR-126) or negative control (lenti-NC). RT-qPCR was used to determine the miR-126 level in the serum and retina. Paraffin sections and retinal vasculature were used to determine the extent of retinopathy. The protein content of vascular endothelial growth factor (VEGF) and pigment epithelium-derived factor (PEDF) in the retina was used as an auxiliary measurement of retinopathy. Western blot analysis and immunofluorescence staining were used to measure the expression of polo-like kinase 4 (PLK4) in rat retinal tissue. In vitro, the cells were transfected with miR-126 inhibitor or mimic and treated with the PLK4 inhibitor, CFI-400945 fumarate. RT-qPCR and western blot analysis were used to detect the miR-126 level
\end{abstract}

Correspondence to: Dr Meixia An or Dr Yanli Liu, Department of Ophthalmology, The Third Affiliated Hospital of Southern Medical University, 183 Zhongshan Road West, Tianhe, Guangzhou, Guangdong 510630, P.R. China

E-mail: anmeixia@163.com

E-mail: yanliliu1116@163.com

${ }^{*}$ Contributed equally

Key words: microRNA-126, diabetic retinopathy, polo-like kinase 4 , proliferation, migration and PLK4 expression. Cell proliferation and migration were measured by EdU and Transwell assays. The diabetic rats were found to exhibit downregulated serum and retinal miR-126 levels compared with the non-diabetic rats. The intravitreal delivery of miR-126 alleviated retinopathy and reduced the diabetes-induced upregulation of PLK4 in retinal tissues. Luciferase reporter assays confirmed that PLK4 mRNA was the target of miR-126. In HG-induced HRCECs, transfection with miR-126 mimic increased the miR-126 level, whereas it downregulated that of its downstream target, PLK4, which was opposite to the effects exerted by the miR-126 inhibitor. Furthermore, miR-126 mimic and CFI-400945 fumarate reduced the HG-induced upregulation of PLK4 expression, as well as cell proliferation and migration. On the whole, the findings of the present study demonstrate that miR-126 reduces experimental diabetic retinopathy and suppresses endothelial cell proliferation and migration by targeting PLK4. Thus, miR-126 and CFI-400945 fumarate may be therapeutic targets for DR.

\section{Introduction}

Diabetes mellitus (DM) is a major metabolic disorder that exerts a negative effect on global public health (1). As a particular microvascular complication of diabetes, diabetic retinopathy (DR) can be classified as non-proliferative diabetic retinopathy (NPDR) and proliferative diabetic retinopathy (PDR), involving retinal neovascularization following ischemia (2). DR has become the leading cause of visual impairment in adults in a number of countries and is a major burden on patients and society $(3,4)$. Thus, it is necessary to examine the pathogenesis of DR and identify effective measures for its prevention.

MicroRNAs (miRNAs or miRs) are a class of highly conserved small-molecule non-coding RNAs that regulate gene expression by binding to complementary target sequences of the 3' untranslated regions (3'-UTR) of mRNAs (5). Recently, studies have focused on miRNAs as important regulatory mediators for diabetic microvascular and macrovascular complications (6,7). miR-126 (also known as miR-126-3p), is an endothelial cell-specific miRNA that 
is highly expressed in endothelial cells, such as capillaries and large blood vessels. It plays a critical role in regulating angiogenic signaling and maintaining vascular integrity in endothelial cells (8). A previous study by the authors found that the serum miR-126 level in patients with DR, particularly those with PDR, was significantly lower than that in healthy individuals (9). However, the precise mechanisms of action of miR-126 in DR remain poorly understood.

Polo-like kinase (PLK) is a type of serine/threonine kinase that plays a key regulator in various cellular events during cell divisions, allowing cells to divide and grow normally. From previous research, it was found that miR-126 directly targets PLK4 in hepatocellular carcinoma (10). PLK4 is a unique member of the PLK family and serves as a primary regulator of centrosome duplication and mitotic progression. It has been demonstrated that PLK4 expression is increased in highly proliferating tissues (11).

Thus, it was hypothesized that miR-126 can affect DR by regulating PLK4 expression. In the present study, rat models of STZ-induced diabetes and high glucose (HG)-induced HRCECs were used to explore the role and mechanisms of miR-126 in $\mathrm{DR}$, with an aim of providing potential therapeutic targets.

\section{Materials and methods}

Animals. Male SD rats weighing 200-250 g ( $\mathrm{n}=55)$ were purchased from the Laboratory Animal Centre of Southern Medical University (Guangzhou, China) and housed with free access to food and water in the Medical Research Center of the Third Affiliated Hospital of Southern Medical University. The environment was maintained at $20-26^{\circ} \mathrm{C}$, relative humidity of 40-60\%, a 12-h light/dark cycle, and with natural and mechanical ventilation. All animal experiments were approved by the Animal Ethics Committee of Southern Medical University.

Diabetes induction. The rats were randomly divided into the non-DM (NDM) and diabetic groups (DM). After being allowed to eat freely for 3 days to adapt to their new living environment, the rats in the DM group $(n=35)$ were administered a single intraperitoneal injection of STZ (60 mg/kg; Sigma-Aldrich; Merck KGaA) dissolved in citrate buffer ( $\mathrm{pH} 4.4$ ) to induce diabetes. The same dose of citrate buffer was used for treating the rats in the NDM group $(n=20)$. One week later, the blood glucose level was measured using a blood sample of the tail vein, and values $\geq 16.7 \mathrm{mmol} / \mathrm{l}$ were considered to indicate diabetes. Rat weight and blood glucose levels were assessed once every 2 weeks.

Intravitreal injection. Lentivirus expressing rno-miR-126 (lenti-miR-126, TCGTACCGTGAGTATAATGCG) and negative control oligonucleotide with a scrambled sequence (lenti-NC, TTCTCCGAACGTGTCACGT) were obtained from GenePharma, Inc. At 1 week following the induction of diabetes, rats were randomly grouped and anesthetized with an intraperitoneal injection of $3 \%$ pentobarbital sodium $(45 \mathrm{mg} / \mathrm{kg})$ and then administered an intravitreal injection of $4 \mu \mathrm{l}$ of lentivirus to each of the two eyes using a 33-gauge needle (Hamilton $^{\mathrm{TM}}$, GuangZhou Jetway Biotech Co., Ltd.). Following the injection, levofloxacin hydrochloride eye gel obtained from EBE Pharmaceutical Co., Ltd. was used to prevent infection.
Animal health and behavior were monitored on a daily basis. Of the 55 rats purchased, 1 rat failed to acquire diabetes, 3 rats died following anesthesia during intravitreal injection, and 3 rats died of diabetes. After 3 months, the remaining rats $(n=48)$ were anesthetized with pentobarbital sodium $(45 \mathrm{mg} / \mathrm{kg})$, and approximately 3-4 $\mathrm{ml}$ of blood was drawn from each rat. The rats were then euthanized by cervical dislocation. The eyeballs were removed from the dead rats after confirming the cessation of the heartbeat and breathing, stiff limbs, whitening lips and the disappearance of blinking reflexes.

Cells and cell culture. Human retinal capillary endothelial cells (HRCECs, JNO-121) were purchased from Guangzhou Jennio Biotech Co., Ltd., and cultured in endothelial cell medium (ECM, Sciencell, Inc.) at $37^{\circ} \mathrm{C}$ in a humidified incubator with $5 \% \mathrm{CO}_{2}$. When the HRCECs reached a confluence of $80-90 \%$, they were digested with $0.25 \%$ trypsin without EDTA and sub-cultured. The HRCECs were cultured in normal glucose (NG, $5.5 \mathrm{mM}$ ) or a high concentration of glucose $(\mathrm{HG}, 25 \mathrm{mM})$ in ECM for $48 \mathrm{~h}$ to examine the mechanisms of DR. Cells were then cultured using CFI-400945 fumarate (MedChemExpress, New Jersey, USA) at a concentration of $200 \mathrm{nM}$ for $24 \mathrm{~h}$.

Cell transfection. miR-126 mimic (sense 5'-UCGUACCGU GAGUAAUAAUGCG-3' and antisense 5'-CAUUAUUAC UCACGGUACGAUU-3'), miR-126 inhibitor (5'-CGCAUU AUUACUCACGGUACGA-3') were purchased from Sangon Biotech Co., Ltd. They were separately transfected into HRCECs at a concentration of $50 \mathrm{nM}$ using Lipofectamine 3000 reagent (Life Technologies; Thermo Fisher Scientific, Inc.) as instructed by the manufacturer for $6 \mathrm{~h}$. The cells were then collected for use in subsequent experiments following $24 \mathrm{~h}$ of transfection.

$R N A$ extraction and $R T-q P C R$. Total serum RNA was extracted using the miRNeasy Serum/Plasma kit (Qiagen, Inc.). RNA was extracted from the mouse retinas or HRCECs using RNA isolater Total RNA Extraction Reagent (TRIzol reagent; R401-01, Nanjing Vazyme Medical Technology Co., Ltd.). RT-qPCR was performed using the miRNA 1st Strand cDNA Synthesis kit (by stem-loop), miRNA Universal SYBR qPCR Master Mix, HiScript ${ }^{\circledR}$ II Q RT SuperMix for qPCR and ChamQ ${ }^{\mathrm{TM}} \mathrm{SYBR}^{\circledR}$ qPCR Master Mix (all from Nanjing Vazyme Medical Technology Co., Ltd.). All the above-mentioned reactions were executed according to the protocols supplied by the manufacturer. Relative quantities of miR-126 were normalized to U6, and PLK4 mRNA was normalized to GAPDH. The thermocycling conditions are as follows: A single cycle of initial denaturation at $95^{\circ} \mathrm{C} / 5 \mathrm{~min}$ (for miRNA) or $95^{\circ} \mathrm{C} / 30 \mathrm{sec}$ (for RNA), then 40 cycles of denaturation at $95^{\circ} \mathrm{C} / 10 \mathrm{sec}$, annealing at $56^{\circ} \mathrm{C} / 30 \mathrm{sec}$, and extension at $72^{\circ} \mathrm{C} / 30 \mathrm{sec}$. After the last cycle, the reaction was completed by a melting curve step at $95^{\circ} \mathrm{C} / 15 \mathrm{sec}, 60^{\circ} \mathrm{C} / 60 \mathrm{sec}$, and $95^{\circ} \mathrm{C} / 15 \mathrm{sec}$. The primer sequences were as follows: miR-126 RT, 5'-GTCGTATCCAGTGCAGGGTCCGAGGTA TTCGCACTGGATACGACCGCATT-3' and forward, 5'-CGC GTCGTACCGTGAGTAAT-3'; U6 RT, 5'-GTCGTATCCAGT GCAGGGTCCGAGGTATTCGCACTGGATACGACTTGG CG-3' and forward, 5'-GGAGACACGCAAACGGAAG-3'; human PLK4 forward, 5'-TCCAAGAGGCAGAAGAAAGA 
CC-3' and reverse, 5'-GCTGAGTGACATCGTTCCATTG-3'. All primers and GAPDH primer solutions were purchased from Sangon Biotech (Shanghai) Co., Ltd. Relative gene expression was calculated using the $2^{-\Delta \Delta \mathrm{Cq}}$ method (12). Three independent experiments were carried out.

Western blot analysis. In order to obtain protein samples, the retinas or HRCECs were placed in RIPA buffer with a protease inhibitor (phenylmethanesulfonyl fluoride), crushed using an ultrasonic cell pulverizer, centrifuged $(5,000 \mathrm{x} \mathrm{g} / 2 \mathrm{~min}$ at room temperature) to obtain the supernatant protein, and quantified using a BCA Protein Assay kit (Nanjing KeyGen Biotech. Co., Ltd.). Equivalent amounts of protein $(20 \mu \mathrm{g})$ from each sample were run at $10 \%$ SDS-PAGE and transferred to $0.22 \mu \mathrm{m}$ polyvinylidene fluoride membranes (EMD Millipore). After blocking with 5\% non-fat dried milk solution at room temperature for $2 \mathrm{~h}$, the membranes were first incubated overnight at $4^{\circ} \mathrm{C}$ with polyclonal rabbit anti-vascular endothelial growth factor (VEGF) antibody (A12303, ABclonal; 1:3,000), polyclonal rabbit anti-pigment epithelium-derived factor (PEDF) antibody (A11782, ABclonal; 1:3,000), polyclonal rabbit anti-PLK4 antibody (12952-1-AP, ProteinTech Group, Inc.; $1: 1,000)$ or monoclonal mouse anti- $\beta$-tubulin antibody (CW0098, ComWin Biotech; 1:5,000), and then with appropriate peroxidase-labeled secondary anti-rabbit or anti-mouse antibodies (RM3002, or RM3001, Beijing Ray Antibody Biotech; 1:3,000) at room temperature for $1 \mathrm{~h}$. Chemiluminescent signals were visualized using Ncm-ECL Ultra (New Cell \& Molecular Biotech Co., Ltd.), while ImageJ software was used to determine the gray strip values.

Paraffin sections and histological assessment. Rat enucleated eyeballs separated from the anterior segments were immediately fixed in $4 \%$ paraformaldehyde solution for $24 \mathrm{~h}$, transferred to gradient dehydration (from 50 to $100 \%$ ethanol), made transparent in xylene, embedded in paraffin, divided into 4- $\mu \mathrm{m}$-thick slices, and attached to slides. Following deparaffinization and rehydration (xylene I, xylene II, $100 \%$ ethanol I, $100 \%$ ethanol II each for $10 \mathrm{~min}$, and 95, 90, 80 and $70 \%$ ethanol each for $5 \mathrm{~min}$ ), the vertical paraffin sections around optic nerve (20 to $60 \mu \mathrm{m}$ apart) were stained with hematoxylin for $3 \mathrm{~min}$ and with eosin for $30 \mathrm{sec}$ (H\&E, Beijing Solarbio Science \& Technology Co., Ltd.) at room temperature and then examined under a light microscope (Olympus Corporation).

Preparation of retinal vasculature by Trypsin digestion. The retinal vasculature was prepared according to the protocol described in the study by Chou et al (13). A fluorescence microscope (Carl Zeiss AG) was used to obtain images of the prepared retinal vessels, and 5 images were selected from each group. The number of endothelial cells and pericytes from these images were counted manually, and the ratio (E/P) was then calculated to determine the extent of retinopathy.

Immunofluorescence staining. The steps for the preparation of the paraffin-embedded sections were the same as those described above. Following deparaffinization and rehydration (xylene I, xylene II, 100\% ethanol I, 100\% ethanol II each for $10 \mathrm{~min}$, and 95, 90, 80 and $70 \%$ ethanol each for $5 \mathrm{~min}$ ), the sections were incubated in $0.01 \%$ trypsin (Beijing Solarbio
Science \& Technology Co., Ltd.) for antigen retrieval at $37^{\circ} \mathrm{C}$ for $15 \mathrm{~min}$, rinsed 3 times with $0.01 \mathrm{M}$ PBS for $5 \mathrm{~min} /$ time, blocked with standard goat serum blocking solution (Wuhan Boster Biological Technology, Ltd.) for $1 \mathrm{~h}$ at room temperature, and incubated overnight at $4^{\circ} \mathrm{C}$ with monoclonal rabbit anti-CD34 (ab81289, Abcam, 1:200) or polyclonal rabbit anti-PLK4 (12952-1-AP, ProteinTech Group, Inc., 1:150). The sections were then incubated for $1 \mathrm{~h}$ at room temperature with donkey anti-rabbit IgG $(\mathrm{H}+\mathrm{L})$ highly cross-adsorbed secondary antibody, Alexa Fluor 594 (A21207, Thermo Fisher Scientific, Inc.; 1:200) antibody and covered with ProLong ${ }^{\mathrm{TM}}$ Diamond Antifade Mountant with DAPI (Thermo Fisher Scientific, Inc.). Images of these sections were obtained using a fluorescence microscope (Carl Zeiss AG).

Dual-luciferase activity assay. PCR was used to amplify the wild-type (Wt), and mutant (Mut) fragments of the PLK4 3'UTR containing the predicted miR-126-binding site. To construct luciferase reporter gene vectors, the target fragments were inserted into the pGL3-Promotor (Promega Corporation) by Nanjing Zhifan Biotechnology Co., Ltd. The constructs were co-transfected into HRCECs along with miR-126 mimic $(50 \mathrm{nM})$ and negative control mimic $(50 \mathrm{nM})$ using Lipofectamine 3000 reagent (Life Technologies; Thermo Fisher Scientific, Inc.) according to the manufacturer's instructions. The sequences were as follows: miR-126 mimic (sense, 5'-UCGUACCGUGAGUAAUAAUGCG-3' and antisense, 5'-CAUUAUUACUCACGGUACGAUU-3'), negative control mimic (sense, 5'-UUCUCCGAACGUGUCACGUTT-3' and antisense, 5'-ACGUGACACGUUCGGAGAATT). Following $48 \mathrm{~h}$ of transfection, the Dual-Luciferase Reporter Assay System (Promega Corporation) was used following the instructions provided by the manufacturer to determine the luciferase activity compared with Renilla luciferase of the samples.

$E d U$ assay. HRCECs were inoculated into $15 \mathrm{~mm}$ glass-bottomed dishes. They were treated separately with different interventions after being adherent and achieving a confluence of $60-70 \%$. Following $48 \mathrm{~h}$ of treatment, the cell proliferative capacity was analyzed with a kFluor488-EdU assay kit (KGA337, Nanjing KeyGen Biotech. Co., Ltd.) following the instructions provided by the manufacturer. In detail, samples were incubated at $37^{\circ} \mathrm{C}$ for $2 \mathrm{~h}$ with a working solution of $50 \mu \mathrm{M}$ EdU. The nuclei were stained blue with Hoechst 33342, protected from light at room temperature for $30 \mathrm{~min}$. The cells were imaged using a fluorescence microscope (Olympus Corporation). A total of 5 fields of view were selected randomly to calculate the positive rate.

Transwell assay. Following treatment of the HRCECs with different interventions for $48 \mathrm{~h}, 1 \times 10^{5} / \mathrm{ml}$ cells were diluted with serum-free ECM and transferred into the upper chamber of a Transwell insert (Costar; Corning, Inc.). An ECM containing 5\% FBS was added to the lower chamber. The non-migrating cells were removed gently from the upper chamber following $24 \mathrm{~h}$ of incubation in a $\mathrm{CO}_{2}$ incubator at $37^{\circ} \mathrm{C}$. Cells that had already migrated through the membrane were fixed with $4 \%$ paraformaldehyde for $20 \mathrm{~min}$ and stained with a solution of $0.5 \%$ crystal violet (Beijing Solarbio Science \& Technology Co., Ltd.) at room temperature for $10 \mathrm{~min}$. The migrated cells were imaged using an 
A

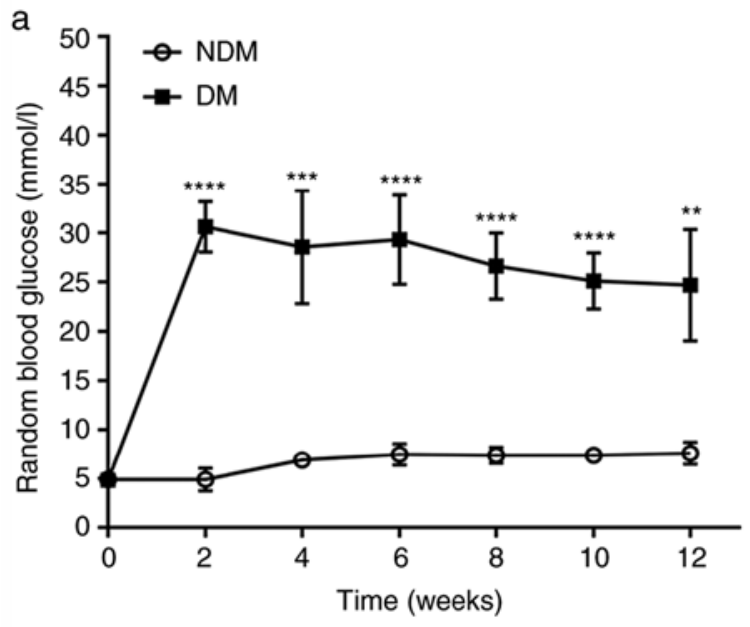

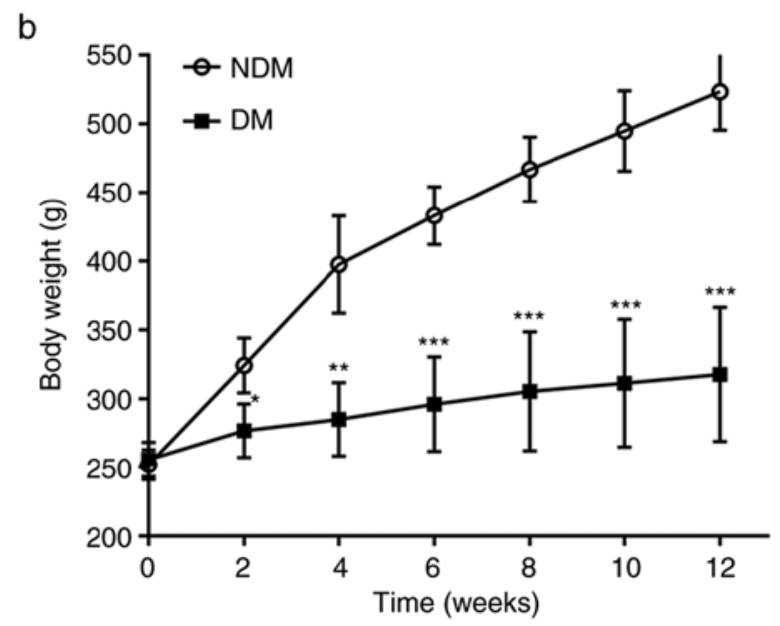

b

B

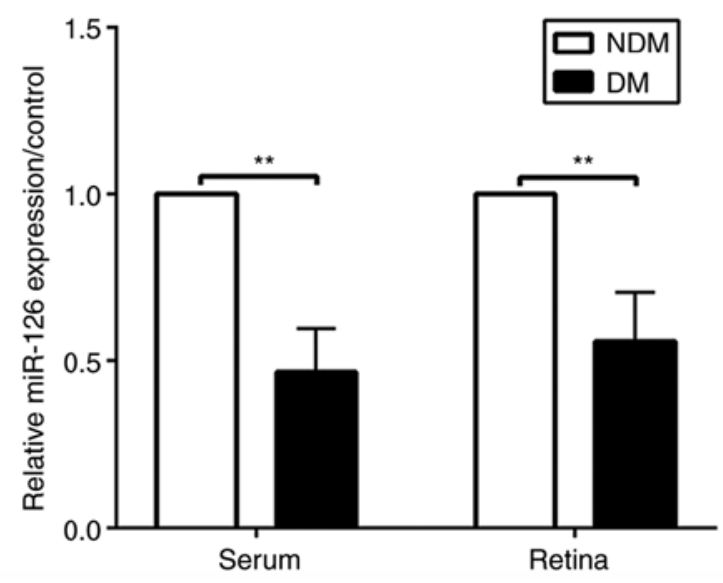

Figure 1. MicroRNA-126 level in serum and retina is downregulated in rats with STZ-induced diabetes compared to non-diabetic rats (A-a). Random blood glucose levels in rats; (A-b). Bodyweight in rats. (B) The miR-126 level in serum and retinas of rats after 3 months of diabetes induction ( $\mathrm{n}=6 / \mathrm{group}$ ). DM, diabetes mellitus; NDM, non-diabetes mellitus. ${ }^{* *} \mathrm{P}<0.01 ;{ }^{* * *} \mathrm{P}<0.001 ;{ }^{* * * *} \mathrm{P}<0.0001$ vs. NDM group.

inverted optical microscope (Olympus Corporation) and 5 fields of view were randomly selected to count the cell numbers.

Statistical analysis. The experimental data are represented as the means \pm SD. Significant differences between 2 groups were evaluated with a t-test using GraphPad Prism 7.0; one-way ANOVA and Tukey's test following ANOVA were used to evaluated the differences between $\geq 3$ groups for multiple comparisons. A value of $\mathrm{P}<0.05$ was considered to indicate a statistically significant difference.

\section{Results}

miR-126 is downregulated in serum and retinas of rats with STZ-induced diabetes. At the established time points of 2 , $4,6,8,10$ and 12 weeks following the induction of diabetes, blood glucose levels in the rats with DM were higher than those in the NDM group (both $\mathrm{P}<0.05$, Fig. 1A-a), while the bodyweight of the rats with DM was lower than that of the rats in the NDM group (both $\mathrm{P}<0.05$, Fig. 1A-b). Random levels of blood glucose in the rats with DM were $>16.7 \mathrm{mmol} / \mathrm{l}$ and did not return to the normal concentration of glucose, proving the successful induction of diabetes. The results of RT-qPCR revealed that the miR-126 level in the serum and retinas was decreased in the diabetic rats at 12 weeks, as compared with the rats in the NDM group (both $\mathrm{P}<0.05$, Fig. 1B).

Intravitreal injection of lenti-miR-126 increases the level of miR-126 without affecting blood glucose levels and weight of the diabetic rats. The lenti-miR126 or its negative control lenti-NC were injected intravitreally into the eyes of rats in the DM and NDM groups at 1 week following the induction of diabetes. Blood glucose levels in the rats with DM injected with lenti-NC (DM+NC) were higher than those in the rats in the NDM group injected with lenti-NC (NDM+NC) at 2, $4,6,8,10$ and 12 weeks following the induction of diabetes. The body weight of the rats in the $\mathrm{DM}+\mathrm{NC}$ group was, however, lower than that of the rats in the NDM+NC group (both $\mathrm{P}<0.05$ ). No significant differences in body weight and blood glucose levels were observed between the rats with DM injected with lenti-miR-126 (DM+miR-126) and the rats in the DM+NC group, suggesting that the intravitreal injection of lentivirus did not affect the physical conditions of rats, as presented in Fig. 2A. At 3 months after the STZ injection, the retinas were harvested and RT-qPCR was performed. The results revealed a decrease in both serum and retinal miR-126 
A
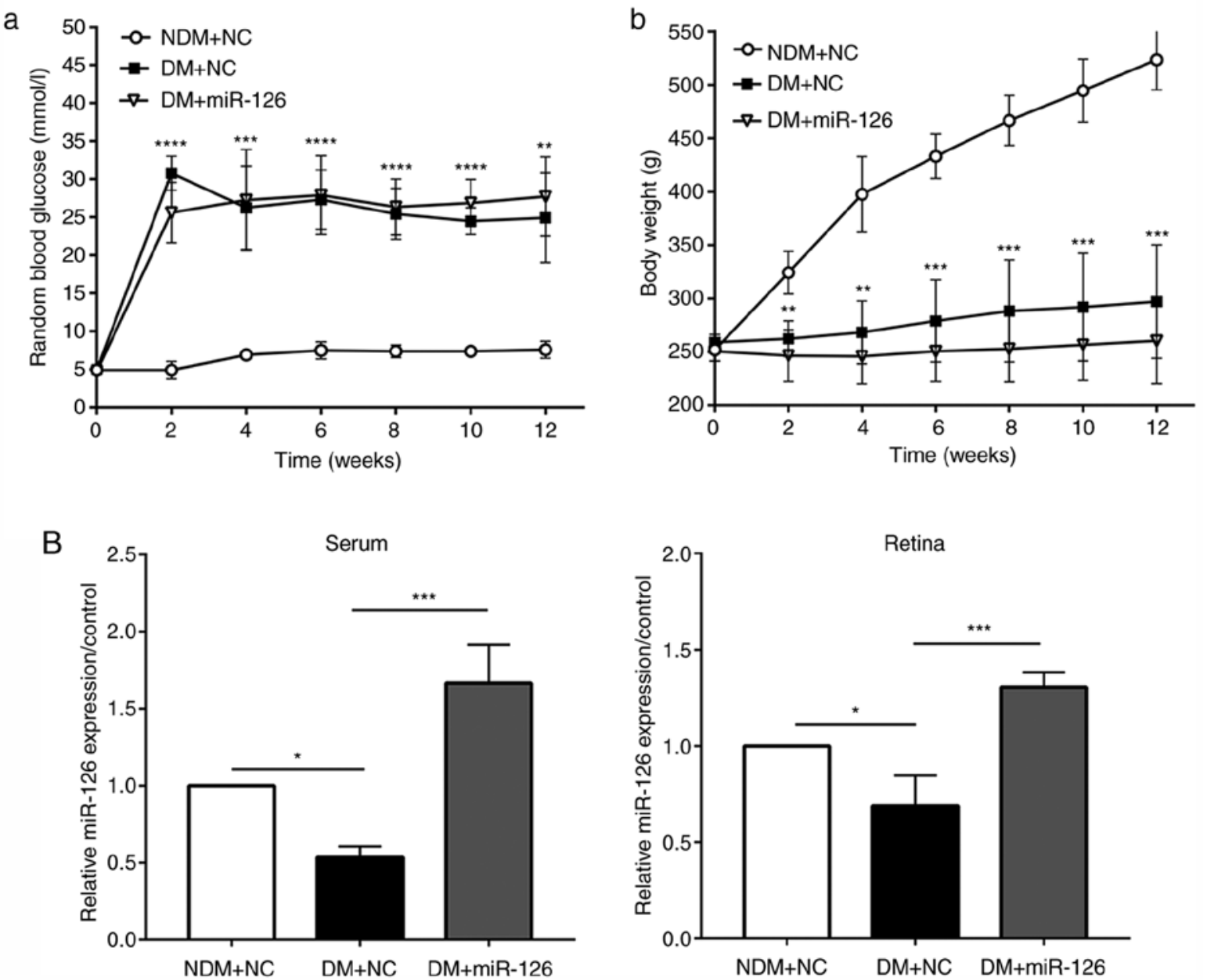

Figure 2. Intravitreal injection of lenti-miR-126 increases the miR-126 expression level of serum and retina in diabetic rats: (A-a). Random blood glucose levels in rats; (A-b). Bodyweight in rats; (B) miR-126 level of serum and retina in rats, which were intravitreally injected with lentivirus (n=6/group). NDM+NC, non-diabetic rats injected with lenti-NC; DM+NC, diabetic rats injected with lenti-NC; DM+miR-126, diabetic rats injected with lenti-miR-126. "P $<0.05$; ${ }^{* *} \mathrm{P}<0.01 ;{ }^{* * *} \mathrm{P}<0.001 ;{ }^{* * * *} \mathrm{P}<0.0001$ vs. NDM+NC or as indicated.

levels in the rats in the $\mathrm{DM}+\mathrm{NC}$ group compared to those in the NDM+NC group. As shown in Fig. 2B, in the serum and retinas of the rats with DM injected with lenti-miR-126, the level of miR-126 was upregulated as compared with the rats with DM injected with lenti-NC (both $\mathrm{P}<0.05$ ).

Intraocular delivery of miR-126 alleviates retinopathy in rats with diabetes. H\&E staining revealed a typical standard architecture of retinal layers arranged regularly in the rats in the NDM group injected with lenti-NC (NDM+NC). However, in the rats with DM injected with lenti-NC, the structures of the ganglion cell layer (GCL), inner nuclear layer (INL) and outer nuclear layer (ONL) were loosened and disordered compared with those in the rats with DM injected with lenti-miR-126 (DM+miR-126) (Fig. 3A). As also shown in Fig. 3A, the rats in the DM+NC group also appeared to have more blood vessels (black arrow) compared with those in the $\mathrm{NDM}+\mathrm{NC}$ and DM+miR-126 groups.

As shown by the retinal vasculature, the nucleus of endothelial cells was large and oval-shaped, parallel to the vasculature in the long axis (white arrows); the nuclei of pericytes were small and triangle-shaped, located at one side of the capillary (black arrows). In the rats in the DM+NC group, the ratio of the number of endothelial cells to the number of pericytes $(\mathrm{E} / \mathrm{P})$ was significantly higher compared to that in the $\mathrm{NDM}+\mathrm{NC}$ and $\mathrm{DM}+\mathrm{miR}-126$ groups $(\mathrm{P}<0.05)$. Furthermore, the retinas from the rats in the $\mathrm{DM}+\mathrm{NC}$ group exhibited more acellular capillaries (triangles) than those from the rats in the NDM+NC and DM+miR-126 groups (Fig. 3B). Immunofluorescence staining also revealed that the retinas from the rats in the DM+NC group exhibited prominent CD34 staining compared to those from the rats in the NDM+NC and DM+miR-126 groups (Fig. 3C).

The results obtained from western blot analysis are depicted in Fig. 3D. It was found that the content of retinal VEGF protein was higher in the rats in the $\mathrm{DM}+\mathrm{NC}$ group than those in the NDM+NC group and lower in the rats in the $\mathrm{DM}+\mathrm{miR}-126$ compared with the rats in the $\mathrm{DM}+\mathrm{NC}$ group. Furthermore, the PEDF retinal content was lower in the rats in the $\mathrm{DM}+\mathrm{NC}$ group than in those in the $\mathrm{NDM}+\mathrm{NC}$ group, while it was higher in the rats in the DM+miR-126 group compared with the rats in the $\mathrm{DM}+\mathrm{NC}$ group. 
A

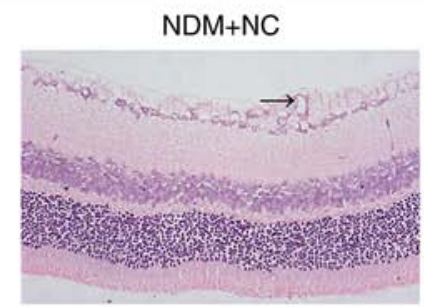

$\mathrm{DM}+\mathrm{NC}$



DM+miR-126

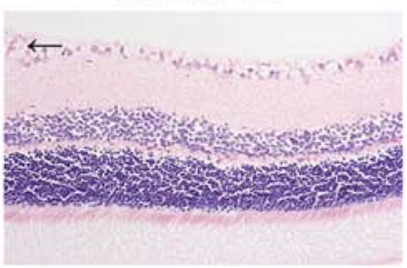

B
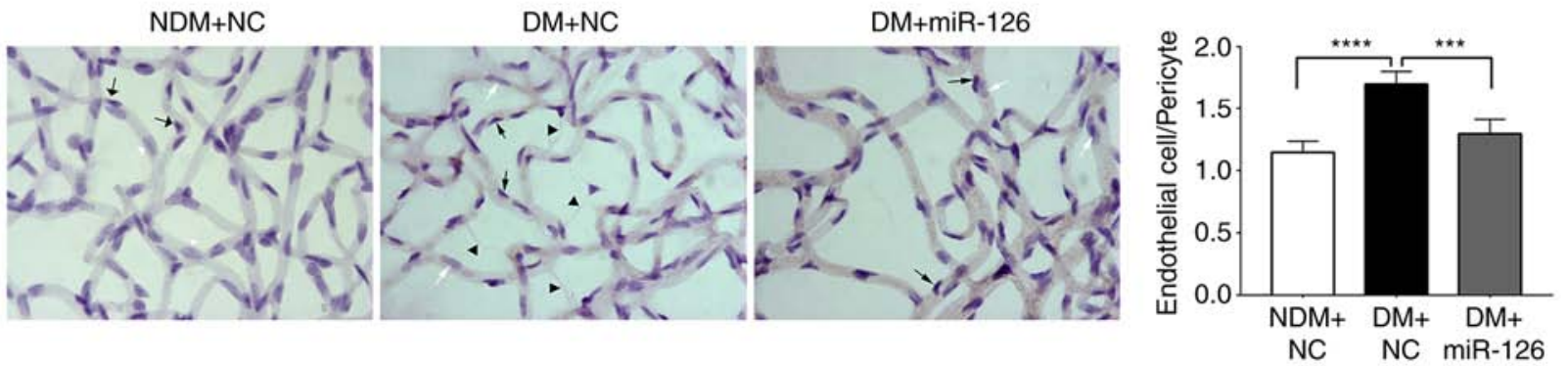

C
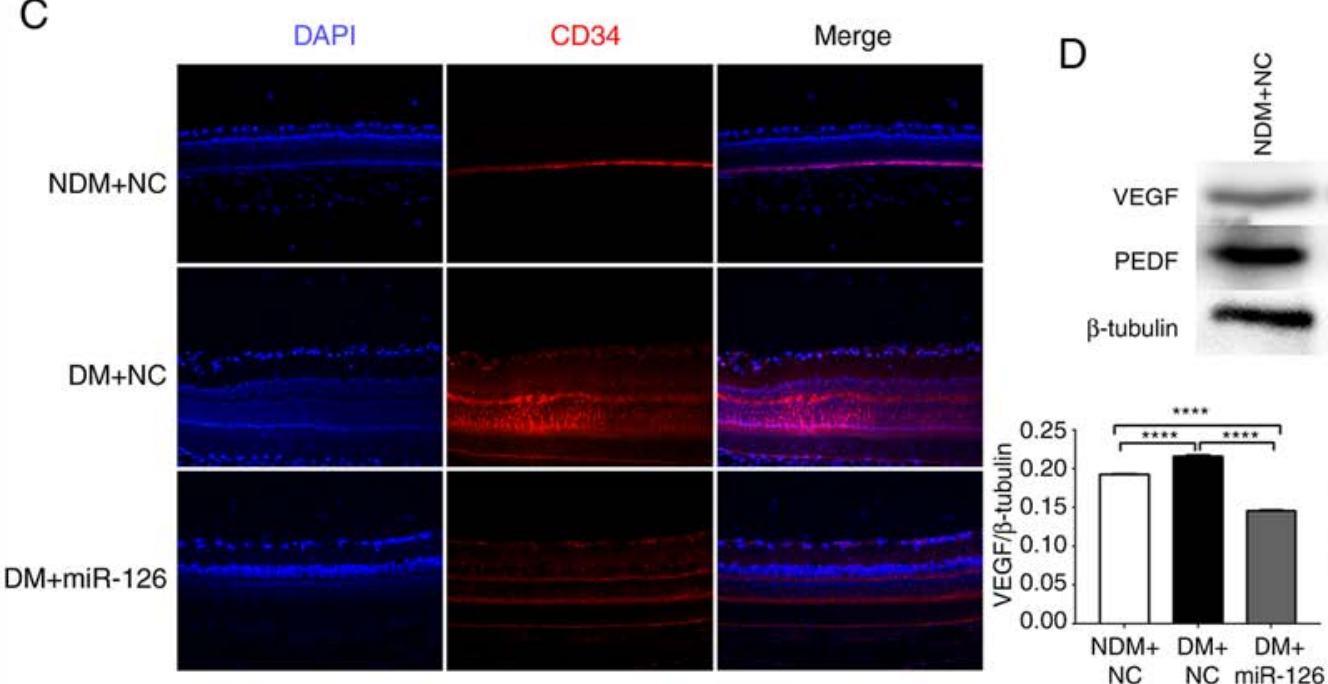




Figure 3. Intraocular delivery of miR-126 alleviates experimental diabetic retinopathy in diabetic rats. (A) H\&E staining of retinal paraffin-embedded sections (magnification, x200). ILM, inner limiting membrane; GCL, ganglion cell layer; INL, inner nuclear layer; ONL, outer nuclear layer; PEL, pigment epithelial layer. (B) The prepared retinal vasculature by trypsin digest (magnification, $\mathrm{x} 400$ ). Endothelial cells are indicated by white arrows, pericytes are indicated by black arrows and acellular capillaries are indicated by triangles. (C) Retinal immunofluorescence staining for CD34. (D) The protein contents of VEGF and PEDF in the rat retina. VEGF, vascular endothelial growth factor; PEDF, pigment epithelium-derived factor; NDM+NC, non-diabetic rats injected with lenti-NC; DM+NC, diabetic rats injected with lenti-NC; DM+miR-126, diabetic rats injected with lenti-miR-126. ${ }^{*} \mathrm{P}<0.05 ;{ }^{* * * *} \mathrm{P}<0.001 ;{ }^{* * * * *} \mathrm{P}<0.0001$.

Intraocular delivery of miR-126 downregulates PLK4 expression in the retinas of diabetic rats. The results of western blot analysis revealed that the protein content of PLK4 in the rats with DM injected with lenti-NC (DM+NC) was higher than that in the rats in the NDM group injected with lenti-NC (NDM+NC) (Fig. 4A). The intravitreal injection of lenti-miR-126 downregulated PLK4 expression in the retinas of the rats with DM compared those of the rats injected with lenti-NC (Fig. 4A). In addition, immunofluorescence staining demonstrated that the retinas of the rats in the $\mathrm{DM}+\mathrm{NC}$ group exhibited prominent PLK4 staining compared to the those of the rats in the $\mathrm{NDM}+\mathrm{NC}$ and $\mathrm{DM}+\mathrm{miR}-126$ groups (Fig. 4B).

miR-126 negatively regulates the expression of PLK4 in $H G$-induced HRCECs. In order to confirm that miR-126 targets PLK4, luciferase reporter assays were performed in which the Wt or Mut PLK4 3'-UTR bound with miR-126 and was fused to the luciferase gene (Fig. 5A). Luciferase reporter assay revealed that transfection with miRNA-126 mimic effectively inhibited the luciferase activity in HRCECs expressing the Wt reporter, but not in those expressing the Mut (Fig. 5B). Furthermore, the results of RT-qPCR indicated that the miR-126 level in HG-induced HRCECs was lower than that of the standard control $(\mathrm{P}<0.05)$. The miR-126 level was reduced $(\mathrm{P}<0.05)$ following transfection of the HG-induced HRCECs with a miR-126 inhibitor. By contrast, transfection with miR-126 mimic increased the miR-126 level $(\mathrm{P}<0.0001)$ (Fig. 5C). As shown in Fig. 5D, in the HG-induced HRCECs, the mRNA expression of PLK4 was upregulated compared with the normal control $(\mathrm{P}<0.05)$. Transfection with miR-126 inhibitor upregulated PLK4 expression $(\mathrm{P}<0.001)$; however, transfection with miR-126 mimic downregulated PLK4 
A

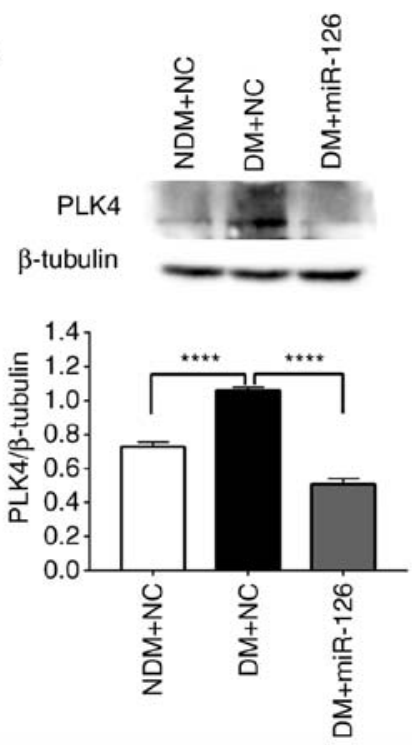

B


Figure 4. Intraocular delivery of miR-126 suppresses the diabetes-induced upregulation of PLK4 expression in the diabetic rat retina. (A) PLK4 protein content in the retina of rats. (B) Retinal immunofluorescence staining for PLK4. PLK4, polo-like kinase 4; NDM+NC, non-diabetic rats injected with lenti-NC; $\mathrm{DM}+\mathrm{NC}$, diabetic rats injected with lenti-NC; DM+miR-126, diabetic rats injected with lenti-miR-126. ${ }^{* * * *} \mathrm{P}<0.0001$.

A

$\begin{array}{rr}\text { Wt PLK4 3'UTR } & \text { 5'UUUUUGAAGAUGUAAAUAAUG } \\ \text { miR-126 } & \text { 3'GCGCAUGGUUUUCAUUAUUAC } \\ \text { Mut PLK4 3'UTR } & \text { 5'UUUUUGAAGAUGUAGGAUCG }\end{array}$

$\mathrm{B}$

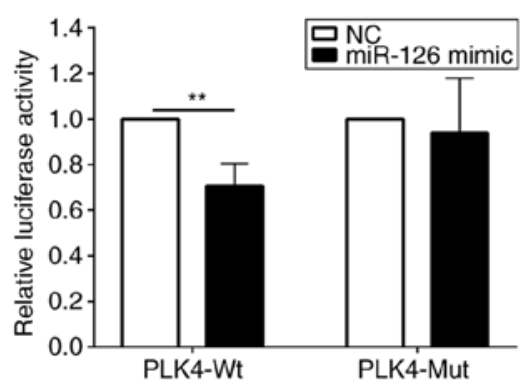

E
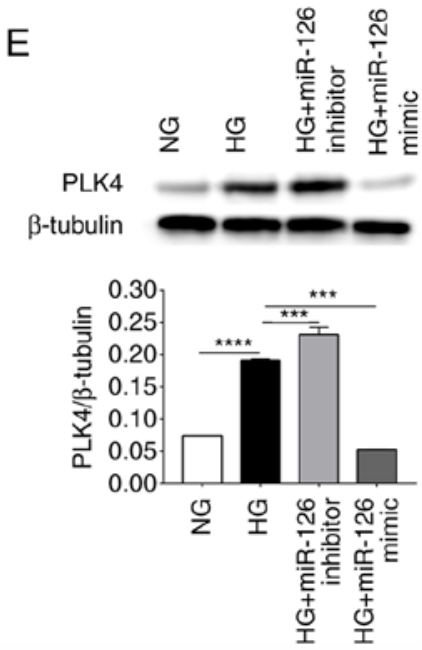

Figure 5. MicroRNA-126 negatively regulates PLK4 expression in high glucose-induced HRCECs. (A) The predicted binding site of miR-126 on PLK4 3'UTR; (B) Luciferase activity of wild-type (Wt) and mutant (Mut) PLK4 3'UTR in each group. (C) Expression level of miR-126 in cells transfected with miR-126 mimic or inhibitor. (D) Expression level of PLK4 mRNA in cells transfected with miR-126 mimic or inhibitor. (E) PLK4 protein content in cells transfected with miR-126 mimic or inhibitor. PLK4, polo-like kinase 4; NG, normal glucose; HG, high glucose. ${ }^{*} \mathrm{P}<0.05 ;{ }^{* *} \mathrm{P}<0.01 ;{ }^{* * *} \mathrm{P}<0.001 ;{ }^{* * * * *} \mathrm{P}<0.0001$.

expression $(\mathrm{P}<0.05)$. The results of western blot analysis revealed that the PLK4 protein content was higher in the HG-induced HRCECs compared to the standard control and was negatively regulated by miR-126 (Fig. 5E).
Overexpression of miR-126 suppresses the proliferation and migration of HG-induced HRCECs by inhibiting PLK4 expression.miR-126 mimic was used to transfect HG-induced HRCECs in order to examine the role of miR-126 in the angiogenesis of 

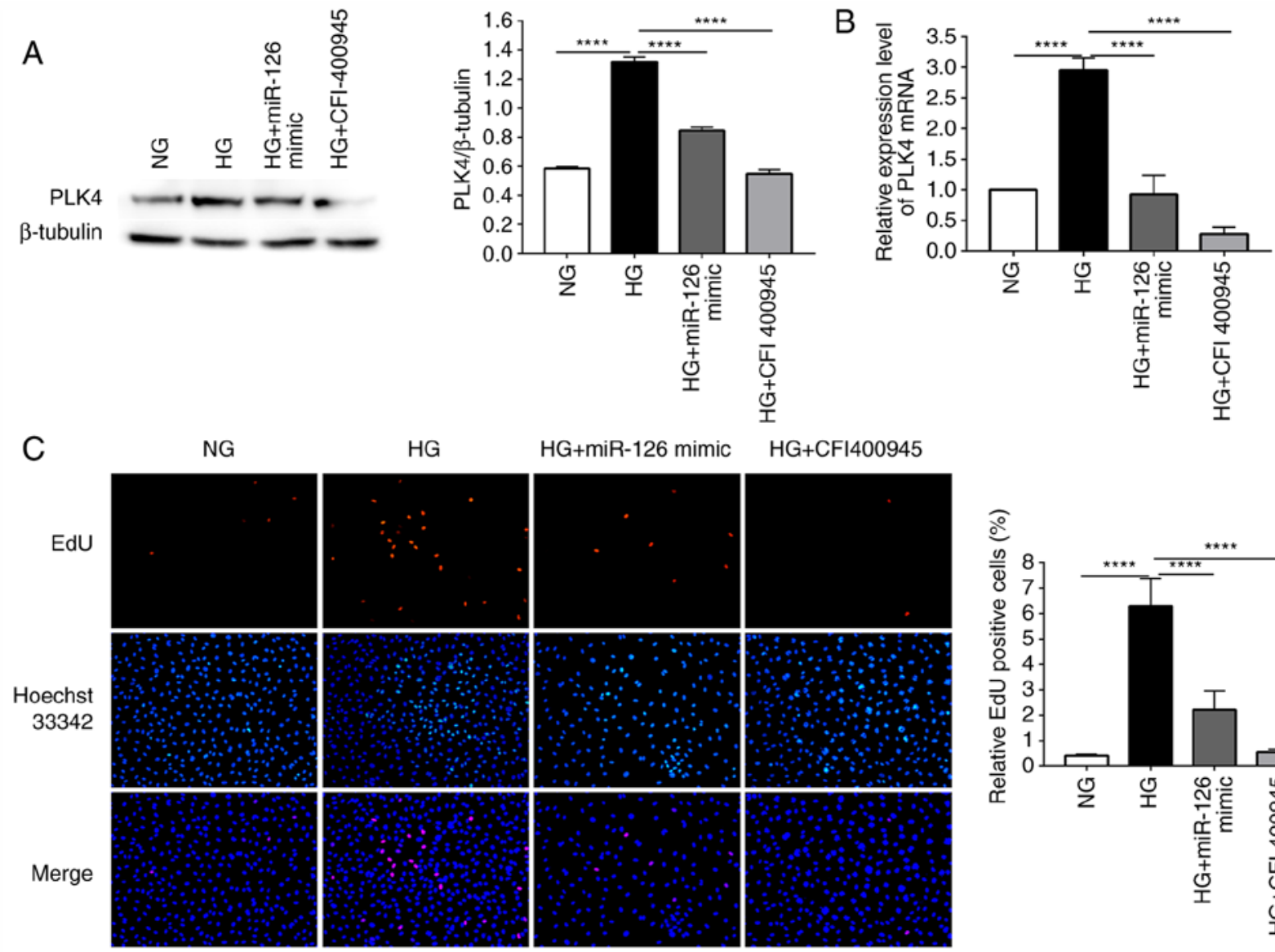

HG
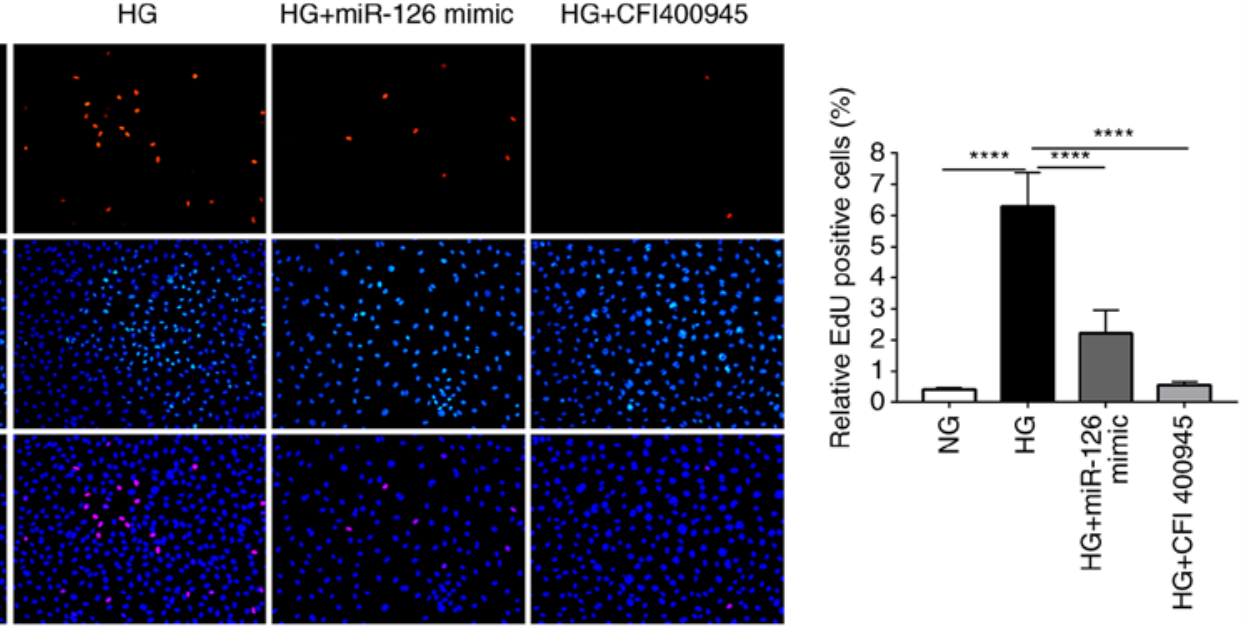

D


Figure 6. miR-126 mimic and CFI-400945 fumarate suppress the high glucose induced-increase in cellular proliferation and migration ability in HRCECs. (A) PLK4 protein content in cells. (B) Expression level of PLK4 mRNA in cells. (C) Proliferative ability of cells is evaluated by EdU assay. (D) Migratory ability of cells was evaluated by Transwell assay. PLK4, polo-like kinase 4; NG, normal glucose; HG, high glucose; CFI-400945 fumarate, a PLK4 inhibitor; EdU, The 5-ethynyl-2'-deoxyuridine. ${ }^{* * * *} \mathrm{P}<0.0001$.

DR. EdU and Transwell assays were used to evaluate the cell proliferative and migratory abilities. CFI-400945 fumarate, a PLK4 inhibitor, was added to the HRCECs to inhibit the expression of PLK4. The results of western blot analysis revealed that the PLK4 protein content was higher in the HG-induced HRCECs than in the NG group. Transfection with miR-126 mimic and treatment with CFI-400945 fumarate suppressed the HG-induced upregulation of PLK4 protein expression (Fig. 6A). In the HG-induced HRCECs, the PLK4 mRNA level was upregulated compared to the NG control, whereas it was downregulated by transfection with miR-126 mimic or treatment with CFI-400945 fumarate (Fig. 6B). In the EdU assay, HG treatment was shown to result in a significant increase in cell proliferation compared to the NG group. At the same time, miR-126 mimic, or CFI-400945 fumarate suppressed the HG-induced increase in cell proliferation (Fig. 6C). In the Transwell assay, HG was shown to increase the migratory ability of the HRCECs, which was suppressed by transfection with miR-126 mimic or treatment with CFI-400945 fumarate (Fig. 6D).

\section{Discussion}

Rat models of STZ-induced diabetes have frequently been used in studies related to diabetes and the associated 
complications (14-16). In the present study, a rat model of STZ-induced diabetes was established. RT-qPCR revealed that both serum and retinal miR-126 levels in the diabetic rats were decreased compared to those in the rats in the NDM group, which is consistent with the findings of a previous study on the human body by the authors (9). Moreover, miRNAs are usually packaged in microvesicles to protect against endogenous RNase activity in the circulation and are present in this stable form. Thus, the serum miR-126 level can reflect its level in the retinal tissues and act as a biomarker for DR screening.

The activation of the angiogenesis process in endothelial cells is essential for normal embryonic development and physiological angiogenesis in adults. However, the formation of new blood vessels in cancer and DR is both pathological and harmful. It has been indicated that miRNAs participate in the maintenance of vascular homeostasis and the prevention of terminal organ sequelae caused by diabetes (17). miR-126 is the only miRNA known to be expressed explicitly in endothelial cell lineage, hematopoietic progenitor cells, and endothelial cell lines (18).

Lentivirus vectors can be stably integrated into the host cell genome for effective exogenous gene expression without any alterations of the cloned gene and have a high gene transduction efficiency. In the present study, lenti-miR-126 was injected intravitreally into the eyes of rats with DM to investigate its function in DR. It was found that the intravitreal injection of lenti-miR-126 increased the miR-126 level in the serum and retinas of rats. Pericytes play a vital role in angiogenesis and vessel stabilization. The condition of reduced retinal pericyte coverage is a well-established fact of DR pathology (19). In the retinal vasculature, the ratio of the number of endothelial cells to pericytes $(\mathrm{E} / \mathrm{P})$ was used to assess $\mathrm{DR}$. The results revealed that the diabetic rats exhibited a higher $\mathrm{E} / \mathrm{P}$ ratio and more acellular capillaries as compared to the rats in the NDM group. However, the intraocular delivery of miR-126 reduced the E/P ratio and acellular capillaries number. In addition, the endothelial marker (CD34) was examined by immunofluorescence to identify capillary endothelium. Although vertical sections around the optic nerve (20 to $60 \mu \mathrm{m}$ apart) were selected for H\&E staining and immunofluorescence, there are still deficiencies in using these methods for the detection of retinopathy. Therefore, the authors intend to make further progress in this matter in future studies.

VEGF is an essential pro-angiogenic growth factor that increases vascular permeability and promotes the proliferation of the vascular endothelial cell. Studies have found that VEGF plays a vital role in the development of DR $(20,21)$. By contrast, PEDF is a potent anti-angiogenic factor with neuroprotective effects, inhibiting the pathological angiogenesis (22). The protein content of VEGF and PEDF in the retina was used as an auxiliary judgment of retinopathy. The results obtained from the present study prove that the intraocular delivery of miR-126 can alleviate retinopathy in diabetic rats. Furthermore, the results of western blot analysis and immunofluorescence staining demonstrated that the intraocular delivery of miR-126 downregulated the diabetes-induced upregulation of PLK4 in vivo, indicating that overexpression of miR-126 may alleviate DR by regulating PLK4 expression.

DR is a microangiopathy in which vascular endothelial cells are the primary cellular targets. Thus, HRCECs were cultured in ECM with a glucose concentration of $25 \mathrm{mM}$ to establish an in vitro DR model and were used to investigate the mechanisms of miR-126 in DR $(23,24)$. Luciferase reporter assay proved that miR-126 could directly bind to PLK4. In HG-induced HRCECs, PLK4 expression was increased compared to the NG group, although the overexpression of miR-126 reduced PLK4 expression. It can thus be concluded that miR-126 negatively regulates PLK4 expression in DR. EdU and Transwell assays were used to investigate whether miR-126 alleviates retinal angiogenesis during the DR process. The miR-126 level was decreased in $\mathrm{HG}$-induced HRCECs, and increased PLK4 expression, as compared to the NG control. Cellular proliferative and migratory abilities were also enhanced. Both miR-126 mimic and CFI-400945 fumarate (a PLK4 inhibitor) reduced PLK4 expression and cellular proliferation and migration. As a result, miR-126 inhibited the proliferation and migration of HG-induced HRCECs by regulating PLK4 expression.

According to the above-mentioned results, miR-126 is decreased in DR and may reduce retinal angiogenesis by targeting PLK4 expression. miR-126 also decreased in tissues and cells of various cancers $(10,25-28)$. It can inhibit proliferation, migration, invasion and cell survival by inhibiting a range of essential target genes, thereby inhibiting tumor progression $(29,30)$. The invention of miRNA agents that are safe, effective, and tolerable in humans would have a positive effect on the proper treatment of DR.

PLK4 has become a potential therapeutic target for various types of advanced cancers (31). CFI-400945 fumarate is a highly selective oral inhibitor of PLK4. Clinical studies have demonstrated that a continuous daily oral dose of CFI-400945 is feasible and safe for patients with advanced solid tumors (32). It was hypothesized that PLK4 inhibitors may play a major role in the treatment of DR. Future studies using rats with DM with CFI-400945 fumarate may, therefore, be performed to confirm the effects of PLK4 during the development of DR and to explore the appropriate therapeutic dose of CFI-400945 fumarate.

In conclusion, the present study found that the serum miR-126 level can act as a distinctive signature for DR screening. The overexpression of miR-126 may inhibit endothelial cell proliferation and migration by directly targeting PLK4 expression, thus alleviating diabetic retinopathy. These results may set new targets for DR screening and treatment.

\section{Acknowledgements}

Not applicable.

\section{Funding}

The present study was supported by the Science and Technology Planning Project of Guangdong Province (grant no. 2014A020212631), and the Science and Technology Planning Project of Guangzhou (grant no. 201604020105).

\section{Availability of data and materials}

The datasets used and analyzed during the current study are available from the corresponding author on reasonable request. 


\section{Authors' contributions}

YaZ, YiL and LW conducted majority of the experiments and assisted with manuscript preparation. HX, ZL, and YX wrote the manuscript and acquired data. WM and LY performed immunofluorescence and analyzed data. DF, YeZ and KK fed the rats and measured their body weight and blood glucose levels. YaL and MA developed the concept, supervised the project, conceived the experiments and critically reviewed the manuscript. All authors read and approved the final manuscript.

\section{Ethics approval and consent to participate}

All animal experiments were approved by the Southern Medical University Committee on the Use and Care of Animals and were performed in accordance with the committee's guidelines.

\section{Patient consent for publication}

Not applicable.

\section{Competing interests}

The authors declare that they have no competing interests.

\section{References}

1. Cho NH, Shaw JE, Karuranga S, Huang Y, da Rocha Fernandes JD, Ohlrogge AW and Malanda B: IDF diabetes atlas: Global estimates of diabetes prevalence for 2017 and projections for 2045 . Diabetes Res Clin Pract 138: 271-281, 2018.

2. Cheung N, Mitchell P and Wong TY: Diabetic retinopathy. Lancet 376: 124-136, 2010.

3. Flaxman SR, Bourne R, Resnikoff S, Ackland P, Braithwaite T, Cicinelli MV, Das A, Jonas JB, Keeffe J, Kempen JH, et al: Global causes of blindness and distance vision impairment 1990-2020: A systematic review and meta-analysis. Lancet Glob Health 5: e1221-e1234, 2017

4. Ting DS, Cheung GC and Wong TY: Diabetic retinopathy: Global prevalence, major risk factors, screening practices and public health challenges: A review. Clin Exp Ophthalmol 44: 260-277, 2016.

5. Lim LP, Lau NC, Garrett-Engele P, Grimson A, Schelter JM, Castle J, Bartel DP, Linsley PS and Johnson JM: Microarray analysis shows that some microRNAs downregulate large numbers of target mRNAs. Nature 433: 769-773, 2005.

6. Giannella A, Radu CM, Franco L, Campello E, Simioni P, Avogaro A, de Kreutzenberg SV and Ceolotto G: Circulating levels and characterization of microparticles in patients with different degrees of glucose tolerance. Cardiovasc Diabetol 16: $118,2017$.

7. Hulsmans M and Holvoet P: MicroRNA-containing microvesicles regulating inflammation in association with atherosclerotic disease. Cardiovasc Res 100: 7-18, 2013.

8. Pishavar E and Behravan J: miR-126 as a therapeutic agent for diabetes mellitus. Curr Pharm Design 23: 3309-3314, 2017.

9. Qin LL, An MX, Liu YL, Xu HC and Lu ZQ: MicroRNA-126: A promising novel biomarker in peripheral blood for diabetic retinopathy. Int J Ophthalmol 10: 530-534, 2017.

10. Bao J, Yu Y, Chen J, He Y, Chen X, Ren Z, Xue C, Liu L, Hu Q, $\mathrm{Li}$ J, et al: MiR-126 negatively regulates PLK-4 to impact the development of hepatocellular carcinoma via ATR/CHEK1 pathway. Cell Death Dis 9: 1045, 2018.

11. Maniswami RR, Prashanth S, Karanth AV, Koushik S, Govindaraj H, Mullangi R, Rajagopal S and Jegatheesan SK: PLK4: A link between centriole biogenesis and cancer. Expert Opin Ther Targets 22: 59-73, 2018.
12. Livak KJ and Schmittgen TD: Analysis of relative gene expression data using real-time quantitative PCR and the 2(-Delta Delta C(T)) method. Methods 25: 402-408, 2001.

13. Chou JC, Rollins SD and Fawzi AA: Trypsin digest protocol to analyze the retinal vasculature of a mouse model. J Vis Exp 76: e50489: 2013.

14. Semkova I, Huemmeke M,Ho MS, Merkl B, Abari E, Paulsson M, Joussen AM and Plomann M: Retinal localization of the glutamate receptor GluR2 and GluR2-regulating proteins in diabetic rats. Exp Eye Res 90: 244-253, 2010.

15. Zhuang P, Muraleedharan CK and Xu S: Intraocular delivery of miR-146 inhibits diabetes-induced retinal functional defects in diabetic rat model. Invest Ophthalmol Vis Sci 58: 1646-1655, 2017.

16. Liu WL: MicroRNA-9 inhibits retinal neovascularization in rats with diabetic retinopathy by targeting vascular endothelial growth factor A. J Cell Biochem 120: 8032-8043, 2018.

17. Zampetaki A, Kiechl S, Drozdov I, Willeit P, Mayr U, Prokopi M, Mayr A, Weger S, Oberhollenzer F, Bonora E, et al: Plasma microRNA profiling reveals loss of endothelial miR-126 and other microRNAs in type 2 diabetes. Circ Res 107: 359-810, 2010.

18. Meister J and Schmidt MHH: miR-126 and miR-126*: New players in cancer. ScientificWorldJournal 10: 2090-2100, 2010.

19. Trost A, Lange S, Schroedl F, Bruckner D, Motloch KA, Bogner B, Kaser-Eichberger A, Strohmaier C, Runge C, Aigner L, et al: Brain and retinal pericytes: Origin, function and role. Front Cell Neurosci 10: 20, 2016.

20. Song E, Dong Y, Han LN, Sui D, Xu Q, Wang X and Wu J: Diabetic retinopathy: VEGF, bFGF and retinal vascular pathology. Chinese Med J (Engl) 117: 247-251, 2004.

21. Ekerbicer N, Gurpinar T, Sisman AR, Guvendi G, Camsari UM and Uysal N: Statins reduce testicular and ocular VEGF: A potential compromise to microcirculation. Microvase Res 119: 60-63, 2018.

22. Simó R, Hernández C; European Consortium for the Early Treatment of Diabetic Retinopathy (EUROCONDOR): Neurodegeneration in the diabetic eye: New insights and therapeutic perspectives. Trends Endocrinol Metab 25: 23-33, 2013.

23. Karthikkeyan G, Nareshkumar RN, Aberami S, Sulochana KN, Vedantham S and Coral K: Hyperglycemia induced early growth response-1 regulates vascular dysfunction in human retinal endothelial cells. Microvasc Res 117: 37-43, 2018.

24. Yao J, Wang J, Yao Y, Wang K, Zhou Q and Tang Y: miR-133b regulates proliferation and apoptosis in high-glucose-induced human retinal endothelial cells by targeting ras homolog family member A. Int J Mol Med 42: 839-850, 2018.

25. Tavazoie SF, Alarcón C, Oskarsson T, Padua D, Wang Q, Bos PD, Gerald WL and Massagué J: Endogenous human microRNAs that suppress breast cancer metastasis. Nature 451: 147-152, 2008.

26. Hua Y, Liang C, Miao C, Wang S, Su S, Shao P, Liu B, Bao M, Zhu J, Xu A, et al: MicroRNA-126 inhibits proliferation and metastasis in prostate cancer via regulation of ADAM9. Oncol Lett 15: 9051-9060, 2018.

27. Chen H, Li L, Wang S, Lei Y, Ge Q, Lv N, Zhou X and Chen C: Reduced miR-126 expression facilitates angiogenesis of gastric cancer through its regulation on VEGF-A. Oncotarget 5: 11873-11885, 2014.

28. Chen M, Peng W, Hu S and Deng J: miR-126/VCAM-1 regulation by naringin suppresses cell growth of human non-small cell lung cancer. Oncol Lett 16: 4754-4760, 2018.

29. Ebrahimi F, Gopalan V, Smith RA and Lam AK: miR-126 in human cancers: Clinical roles and current perspectives. Exp Mol Pathol 96: 98-107, 2014

30. Zhai $\mathrm{X}$ and $\mathrm{Xu} \mathrm{W}$ : Long noncoding RNA ATB promotes proliferation, migration, and invasion in bladder cancer by suppressing microRNA-126. Oncol Res 26: 1063-1072, 2018.

31. Press MF, Xie B, Davenport S, Zhou Y, Guzman R, Nolan GP, O'Brien N, Palazzolo M, Mak TW, Brugge JS and Slamon DJ: Role for polo-like kinase 4 in mediation of cytokinesis. Proc Natl Acad Sci USA 116: 11309-11318, 2019.

32. Veitch ZW, Cescon DW, Denny T, Yonemoto LM, Fletcher G, Brokx R, Sampson P, Li SW, Pugh TJ, Bruce J, et al: Safety and tolerability of CFI-400945, a first-in-class, selective PLK4 inhibitor in advanced solid tumours: A phase 1 dose-escalation trial. Br J Cancer 121: 318-324, 2019.

This work is licensed under a Creative Commons Attribution-NonCommercial-NoDerivatives 4.0 International (CC BY-NC-ND 4.0) License. 